\title{
Trajectories of Body Mass Index from Young Adulthood to Middle Age among Canadian Men and Women
}

\author{
Meng Wang, ${ }^{1}$ Yanqing Yi, ${ }^{1}$ Barbara Roebothan, ${ }^{1}$ Jennifer Colbourne,, \\ Victor Maddalena, ${ }^{1}$ Peizhong Peter Wang, ${ }^{1}$ and Guang Sun ${ }^{2}$ \\ ${ }^{1}$ Division of Community Health \& Humanities, Faculty of Medicine, Memorial University of Newfoundland, \\ St. John's, NL, Canada A1B 3V6 \\ ${ }^{2}$ Discipline of Medicine, Faculty of Medicine, Memorial University of Newfoundland, St. John's, NL, Canada A1B 3V6 \\ Correspondence should be addressed to Yanqing Yi; yanqing.yi@med.mun.ca
}

Received 20 August 2015; Revised 24 October 2015; Accepted 30 November 2015

Academic Editor: Jeanine M. Buchanich

Copyright (C) 2015 Meng Wang et al. This is an open access article distributed under the Creative Commons Attribution License, which permits unrestricted use, distribution, and reproduction in any medium, provided the original work is properly cited.

\begin{abstract}
Knowledge regarding the heterogeneity of BMI trajectories is limited for the Canadian population. Using latent class growth modelling, four distinct BMI trajectories of individuals from young adulthood to middle age were identified for both women and men from the longitudinal data of the National Population Health Survey. The associations between BMI trajectories and the individuals' sociodemographic characteristics and behavioural factors were also examined. Aboriginal women were found more likely to be in the long-term overweight or obese groups. It reveals that increased years of smoking, drinking, and being physically active were associated with lowering the BMI trajectory in all groups for both women and men, with some exceptions in the longterm normal weight group for men. Increased years of rural living, being employed, and living with low income were associated with raising the BMI trajectory in all groups for women and in some groups for men. Food insecurity was associated with raising the BMI trajectory in each group for both women and men.
\end{abstract}

\section{Introduction}

Since 1985, the prevalence of obesity, defined by a BMI of $30 \mathrm{~kg} / \mathrm{m}^{2}$ or higher, has greatly increased in Canada [1]. Excess body weight is associated with premature death [2] and numerous chronic health conditions such as cardiovascular disease and type 2 diabetes [3]. There is a general agreement that the recent obesity epidemic is more strongly associated with environmental changes and negative health behaviours than genetic factors [2]. However, there is no clear consensus on the relative importance of these environmental changes and/or health behaviours in contributing to the obesity epidemic [4]. To better understand the multiple factors associated with obesity, it is important to capture the heterogeneity of BMI changes over time.

Trajectory analyses effectively capture body weight changes over time and can offer new insights into the determinants of obesity $[5,6]$. However, many existing studies that have investigated obesity neither capture weight fluctuations nor detect the trajectory of body weight changes over time. For instance, the majority of previous studies assessed weight status based on a limited number of measurements of body weight or BMI. Typically, cross-sectional studies defined body weight status based on a one-time measurement [7], and many longitudinal studies used a short period of followup and/or assessed the BMI changes based on a limited time frame [8-11]. Additionally, multiple studies have utilized conventional growth modelling (CGM) to model BMI trajectories [12-16]. CGM is an umbrella term for multilevel (hierarchical) modelling [17] and latent curve analysis [18]. CGM uses an average pattern to measure the developmental trajectory for a population, with the assumption that all individuals follow a similar pattern. In addition, it assumes that covariates associated with growth patterns have a similar impact for every individual. Therefore, CGM may only be suited for a process in which the entire population follows a common pattern [19]. However, there may be meaningful subgroups within a population. For example, some people 
will never have a high BMI, some will always have a high BMI, and others will have a BMI that fluctuates with time.

Latent class growth modelling (LCGM) is an effective method for modelling BMI changes. In contrast to CGM, LCGM is a powerful statistical approach that captures the heterogeneity of BMI changes over time. LCGM assumes that there may be several different trajectory groups among population members and focuses on the identification and examination of these different developmental patterns [19-21]. Moreover, generalized LCGM can assess if a risk factor (i.e., a time-constant covariate) can predict membership probability of trajectory groups and whether and by how much a covariate (either time-varying or time-constant) can modify the trajectory within each subgroup [19]. For example, the generalized model can test if differences exist across cohorts in their developmental trajectories (i.e., cohort effects) when multiple cohorts are combined in order to construct an overall trajectory model [19]. Furthermore, by utilizing LCGM, the impact of obesity-related factors on distinct levels of BMI trajectories can be examined [5]. Although LCGM has been used to identify BMI trajectories for youth and children [2224], only three studies applied LCGM to analyze the patterns of BMI or body weight changes on a substantial period of adult life [25-27]. The study by Ostbye et al. investigated four distinct upward-sloping trajectories of BMI in adults aged 18-49 years [25]. Likewise, Finkelstein et al. identified four different BMI trajectory groups with increasing trends for adults aged 25-33 years with class I obesity [26]. Recently, Malhotra et al. studied body weight trajectories of women and men stratified by baseline BMI categories of normal weight, overweight, and classes I, II, and III obesity, separately. Their results showed weight gain variations by and within the BMI categories as well as gender differences in body weight trajectories [27]. However, these studies examined the US population and did not consider some important determinants of obesity such as food insecurity and lifestyle factors including smoking and physical activity. Moreover, the majority of previous research has focused on the associations among sociodemographic characteristics, such as gender, race/ethnicity, age, and educational attainment, and BMI trajectories $[5,22,25,26,28,29]$. Few studies have examined whether behavioural or lifestyle factors, such as smoking, drinking, or physical activity, alter BMI trajectories.

Gender may play an important role in the obesity epidemic through both biological and social factors [30-32]. Some studies suggest that the prevalence of obesity and sociodeterminants of obesity differ by gender [33-36]. For example, Jones-Johnson et al. suggested that the prevalence of obesity was higher among women than men in all racial groups [35]. Previous research also reported that low income was consistently associated with an increased risk of obesity in women but not men [36]. Furthermore, different trajectory patterns of body weight/BMI are found between women and men $[26,27]$. Despite these gender differences, the topic of gender specific factors associated with obesity and gender tailored solutions remain limited [37, 38]. It is of interest to explore the potential gender differences in the BMI trajectories for young to middle-aged adults and investigate the associated factors.
The intent of this study is to apply LCGM to identify the BMI trajectories of Canadian women and men from young adulthood to middle age ( $20-55$ years). Specifically, the objectives are to examine how risk factors such as race/ethnicity, gender, and education level predict the group membership of BMI trajectories and how time-varying covariates, including income status, employment status, duration of physical activity, smoking, drinking, and rural living, modify the identified BMI trajectories. This study also explores the gender differences in these associations.

\section{Methods}

Data from the National Population Health Survey (NPHS) were used in this study. The NPHS is a nationally representative longitudinal health survey on economic, social, demographic, occupational, and environmental correlates of health. It was conducted by Statistics Canada beginning in 1994/1995, and the data were collected biennially thereafter until the most recent survey in 2010/2011. The NPHS is a multistage complex longitudinal survey dataset which followed 17,276 respondents from all ages. The Household component of the NPHS has nine cycles; the overall response rate in cycle one was $83.6 \%$. The longitudinal response rates in subsequent cycles were $92.8 \%, 88.3 \%, 84.9 \%, 80.8 \%, 77.6 \%$, $77.0 \%, 70.7 \%$, and $69.7 \%$ for cycles two to nine, respectively [39]. The NPHS did not include persons living on Aboriginal reserves or Crown Lands, residents of healthcare institutions, full-time members of the Canadian Forces Bases, and some of those who lived in remote areas of Ontario and Québec [39].

To identify the BMI trajectories for young to middle-aged adults, 4790 respondents, aged 20-39 years at baseline and having at least four BMI records in the NPHS, were included in this study. Approximately $17 \%$ of participants had less than four BMI values and were excluded. The cumulative attrition rates to cycle four for the excluded group are $95.8 \%$ and $19.1 \%$ for the included group. This shows that nonresponse is the main reason that respondents had less than four BMI values. No significance was found between the respondents included in the analysis and those excluded by the variables of age, gender, and physical activity level at baseline. However, at baseline, the respondents included differed from those excluded in terms of ethnic/racial background, education level, mean BMI, family income, employment status, rural residence, smoking, alcohol consumption, and food insecurity.

2.1. Basic Trajectory Variables. Age, rather than the calendar year of the survey, was used to define the time variable in this trajectory analysis. An Accelerated Longitudinal Design (ALD) [40] was employed in this study in order to analyze the patterns of BMI change over a period of 36 years even though the NPHS contains only 18 years of collected data for each individual. Previous research has demonstrated that ALD can reasonably and effectively approximate age-related developmental changes over time by linking different cohorts together [41].

BMI was used as the trajectory variable. The NPHS provided up to nine self-reported measures of weight and 
height over a span of 18 years. The NPHS calculated BMI by dividing body weight in kilograms by the square of standing height in metres. The imputed data provided by Statistics Canada was used to determine the available BMI values of respondents. The NPHS imputed incomplete or missing height and weight values first and then recalculated the BMI based on the imputed values of panel members who provided a complete or partial response to interviews conducted from 1994 to 2011 [39]. Detailed information can be provided upon request [39].

2.2. Covariates. The selection of covariates was based on a literature review and determined by information available from the NPHS. Previous studies found that BMI and/or BMI changes are often linked to gender, age, race/ethnicity, education, and income [12, 33, 42-45]. In addition, food insecurity, place of residence, and lifestyle factors, such as physical activity, smoking, and alcohol use, are all potentially associated with body mass or body mass change [44, 46-51]. Covariates can be classified as risk factors (time-invariant) and time-varying covariates.

2.2.1. Risk Factors. Race/ethnicity, defined as Aboriginal or Non-Aboriginal, and educational attainment, defined by graduation from high school, were included as risk factors with the assumption that they have a potential impact on group membership of trajectories.

2.2.2. Time-Varying Covariates. Time-varying covariates (TVCs) include years of smoking, drinking, rural living, being employed, living with low income, and being physically active. "Years of being physically active" was defined based on a derived variable of the physical activity index in the NPHS. This derived variable was categorized into three levels in the NPHS, from active to inactive, based on the average daily energy expended during leisure time activities in the past three months. An energy expenditure of less than $1.5 \mathrm{kcal} / \mathrm{kg} /$ day was classified as inactive since it did not meet the recommended thirty minutes of exercise per day [39]. Respondents were assigned a value of " 1 " if their energy expenditure was $1.5 \mathrm{kcal} / \mathrm{kg} /$ day or greater in $1994 / 95$, or "0" otherwise. For each wave, a value of " 2 " was added to the previous value if the individual was classified as having an energy expenditure of $1.5 \mathrm{kcal} / \mathrm{kg} /$ day or greater in the wave, or " 0 " otherwise. For waves with missing data, the value of "years of being physically active" from the previous wave was used. This method was also used to define all other TVCs.

"Years of smoking" was based on a respondent's current smoking habits at each wave. "Years as a regular drinker" was based on the self-report of the respondent at each wave. "Years of living with low income" was based on total household income adjusted for the number of people living in the household at each wave. The respondents in the lowest or low-middle income categories were considered as living with low income. "Years in employment" was based on a respondent's working status in the past 12 months at each wave. "Years of rural living" was based on the geographical location of the postal code of the household at each wave.
In addition, the effects of age and food insecurity on BMI trajectory was investigated within each identified group. These two variables were included in the model as TVCs [19]. The "age cohort" variable was defined based on a respondent's age at baseline. Those aged 20-29 years in 1994/95 were coded as "1," and those aged 30-39 years in 1994/95 were coded as " $0 . "$ Because of the limited sample size, the impact of age by decade was examined rather than by a single year. The variable "food insecurity" is a latent variable defined from the trajectories of the food insecurity flag variable asked in cycles $2,7,8$, and 9. In the NPHS, the following questions were asked: "In the past 12 months, did you or anyone else in your household worry that there would not be enough to eat because of a lack of money?", "Did you or anyone else in your household not have enough food to eat because of a lack of money?", and "Did you or anyone else in your household not eat the quality or variety of foods that you wanted to eat because of a lack of money?". The NPHS determined that a respondent was food insecure if they answered "yes" to any of the above questions. LCGM was used to identify different food insecurity patterns and then each individual was assigned to the most probable food insecurity trajectory, which formed the latent classes of food secure and food insecure.

2.3. Statistical Analysis. LCGM was used to capture BMI trajectories and examine the associations between the identified trajectories and covariates [52]. All analyses were stratified by gender using SAS version 9.3 (SAS Institute). LCGM uses maximum likelihood methods to estimate model parameters. Maximization was performed using a general quasi-Newton procedure [19].

To determine the optimal number of trajectories, the criteria for building the model were the Bayesian Information Criterion (BIC), the value of group membership probability (GMP), the average posterior probability (AvePP), and the significance of polynomial terms $[19,53]$. Specifically, model selection started with one cubic group and more groups were added if the model with the added groups had a better fit based on the above criteria. Successive models with between two and seven trajectories were tested. For model selection, only the polynomial terms (quadratic or cubic) with significant coefficients were kept before adding additional groups. The linear terms were held whether they were significant or not [53]. For the purpose of representativeness, the GMP of each group was set to be $\geq 5 \%$. Each participant was assigned to the trajectory group for which he or she had the highest posterior probability of membership. Within each trajectory group, the values of average posterior probability of group membership were ascertained [19]. The trajectory analysis was conducted separately for women and men because of the compelling evidence of gender differences in obesity $[5,22$, $25,28,42,54]$.

The generalized LCGM was used to assess if risk factors (race/ethnicity and education) predict the GMP of BMI trajectory groups and to examine whether and by how much TVCs (years of being physically active, smoking, drinking, living with low income, being employed, and rural living), age, and food insecurity modify the trajectory within each 
group. The estimation of the parameters in LCGM and the assessment of the associated factors of trajectories were completed simultaneously in the same LCGM model [19]. Coefficients of risk factors indicate the change of relative odds of following a trajectory group as opposed to the reference group with one unit change in the risk factor. Coefficients of TVCs measure the changes in the BMI trajectory associated with changes in the TVCs at the trajectory level. Coefficients of age or food insecurity measure the changes in the BMI trajectory associated with age or food insecurity. The estimates related to TVCs, age, and food insecurity are trajectory group specific, which can vary across trajectories. This is one of the most important strengths of LCGM [19].

All descriptive analyses were weighted using the survey sampling weights and bootstrap weights which were provided and suggested by Statistics Canada. Within LCGM, no sampling weights were utilized because most variables used in the calculation of sampling weights (e.g., race/ethnicity and place of residence) were included as covariates in the LCGM model. According to previous research, this would make unweighted estimates less biased than weighted estimates [55]. Differences with $P$ value $<0.05$ were considered to be statistically significant.

\section{Results}

Of the 4790 individuals included in this study, $49.9 \%$ were women. They were predominantly Caucasian (88.8\%), with $1.14 \%$ from an Aboriginal population. Most of the respondents had graduated from high school; however, $18.4 \%$ of the individuals had less than a high school education. From 1994 to 2011, the weighted prevalence of being overweight (BMI 25.0-29.9), obese class I (BMI 30.0-34.9), obese class II (BMI 35.0-39.9), and obese class III (BMI $\geq 40.0)$ increased from $31.1 \%$ to $39.0 \%, 8.6 \%$ to $16.7 \%, 1.8 \%$ to $4.4 \%$, and $0.8 \%$ to $2.6 \%$, respectively. Consequently, the weighted percentage of individuals in the underweight (BMI < 18.5) and normal weight (BMI 18.5-25) categories declined from 2.5\% to $0.9 \%$ and from $55.1 \%$ to $36.5 \%$, respectively.

Four BMI trajectory groups, Normal-Stable (N-S), Normal-Overweight (N-OV), Overweight-Obese (OV-OB), and Obese-Up (OB-UP), were identified for both women and men (Figures 1 and 2, Tables 1 and 2). The identified BMI trajectories differ in baseline values of BMI at age of 20 years with increasing values of BMI over time. The average posterior probability of each trajectory group exceeded 0.85 for both women and men.

For women, approximately $40.9 \%$ belonged to the $\mathrm{N}$ $S$ BMI trajectory group. On average, women in this group remained in the normal weight range between the ages of 20 and 55 years. The proportion of underweight and normal weight women in this group was greater than $90 \%$ before age 30 and then decreased to $85 \%$ after the age of 50 years.

The group membership probability (GMP) for the N-OV group of women was $33.7 \%$. The $\mathrm{N}-\mathrm{OV}$ trajectory started in the normal weight range at the age of 20 , with an average BMI of 22.6, then progressed to the overweight range, and remained overweight until age 55. Within the N-OV group,

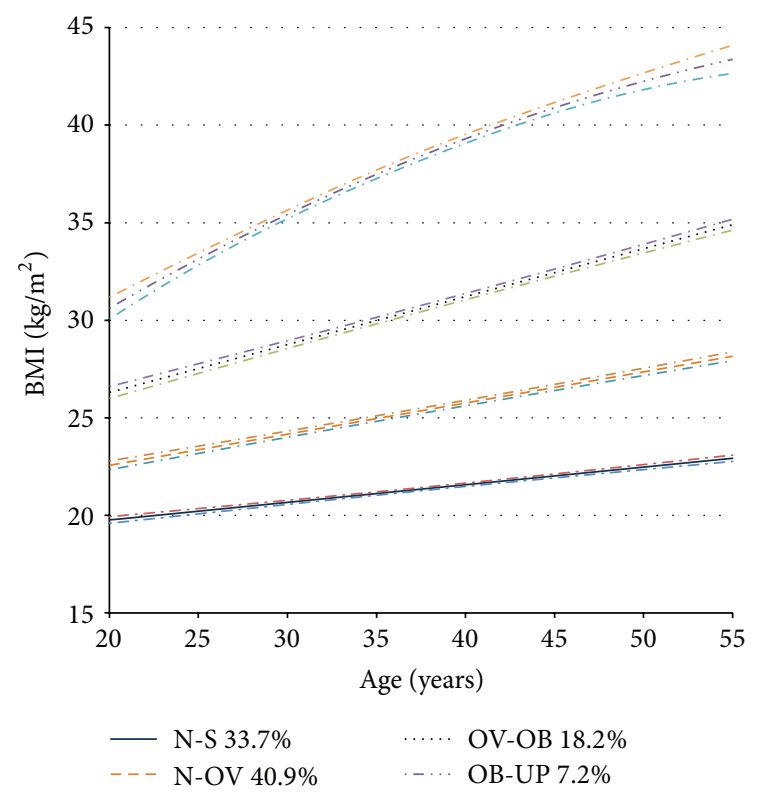

FIgURE 1: BMI trajectories for women (20-55 years), with 95\% confidence intervals (four-group model, no covariates included), NPHS, 1994-2011.

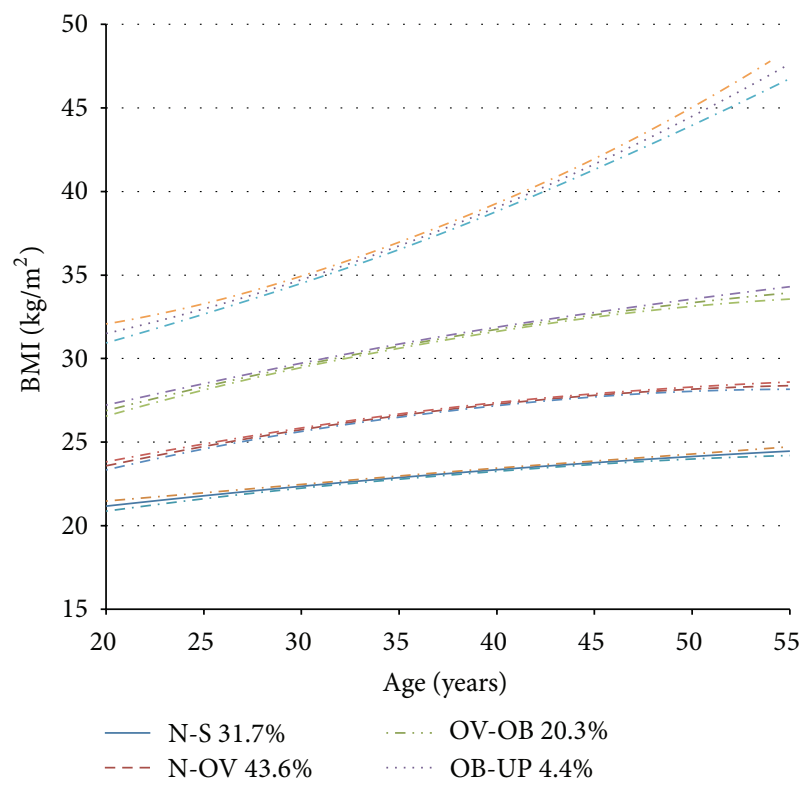

FIGURE 2: BMI trajectories for men (20-55 years), with $95 \%$ confidence intervals (four-group model, no covariates included), NPHS, 1994-2011.

approximately $85 \%$ of the women had a BMI of less than 25.0 by age 30 ; however, most became overweight after age 40 . The proportion of obese women in this group increased steadily after age 40 , but this proportion did not exceed $25 \%$ at any age.

The trajectory of the OV-OB group of women (GMP of $18.2 \%$ ) started at an overweight status at age 20, with an average BMI of 26.4, increased to reach obese class I, and kept increasing but remained in the BMI range of obese class I. 
TABLE 1: Parameters estimated for BMI trajectories for women (20-55 years), NPHS, 1994-2011.

\begin{tabular}{|c|c|c|c|c|c|}
\hline The trajectory of BMI & $\begin{array}{c}\text { Intercept-BMI at age } 20 \\
\text { (s.e.) }\end{array}$ & Linear term (s.e.) & Quadratic term (s.e.) & GMP & AvePP \\
\hline N-S & $19.75(0.16)$ & $0.09(0.004)$ & - & $40.9 \%$ & 0.87 \\
\hline $\mathrm{N}-\mathrm{OV}$ & $22.57(0.21)$ & $0.16(0.005)$ & - & $33.7 \%$ & 0.87 \\
\hline OV-OB & $26.38(0.29)$ & $0.25(0.007)$ & - & $18.2 \%$ & 0.87 \\
\hline OB-UP & $32.42(1.34)$ & $0.71(0.075)$ & $0.005(0.001)$ & $7.2 \%$ & 0.88 \\
\hline
\end{tabular}

$\mathrm{N}-\mathrm{S}$, normal stable group; $\mathrm{N}-\mathrm{OV}$, normal weight to overweight group; OV-OB, overweight to obesity group; OB-UP, obesity-up group; GMP, group membership probability; AvePP, average posterior probability; and s.e., standard error.

TABLE 2: Parameters estimated for BMI trajectories for men (20-55 years), NPHS, 1994-2011.

\begin{tabular}{|c|c|c|c|c|c|}
\hline The trajectory of BMI & $\begin{array}{c}\text { Intercept-BMI at age } 20 \\
\text { (s.e.) }\end{array}$ & Linear term (s.e.) & Quadratic term (s.e.) & GMP & AvePP \\
\hline $\mathrm{N}-\mathrm{S}$ & $21.20(0.66)$ & $0.17(0.03)$ & $0.001(0.0005)$ & $31.7 \%$ & 0.87 \\
\hline $\mathrm{N}-\mathrm{OV}$ & $23.77(0.52)$ & $0.38(0.03)$ & $0.003(0.0004)$ & $43.6 \%$ & 0.88 \\
\hline OV-OB & $26.78(0.77)$ & $0.41(0.04)$ & $0.003(0.0006)$ & $20.3 \%$ & 0.87 \\
\hline OB-UP & $31.61(1.43)$ & $0.04(0.08)$ & $0.006(0.001)$ & $4.4 \%$ & 0.86 \\
\hline
\end{tabular}

$\mathrm{N}-\mathrm{S}$, normal stable group; N-OV, normal weight to overweight group; OV-OB, overweight to obesity group; OB-UP, obesity-up group; GMP, group membership probability; AvePP, average posterior probability; and s.e., standard error.

Approximately $65 \%$ of the women in the OV-OB group were overweight at age 20 , but the prevalence of being overweight, without being obese, was negligible by age 55 . The prevalence of obesity in this group exceeded $70 \%$ after age 45 .

Approximately $7.3 \%$ of women belonged to the OB-UP group. The OB-UP trajectory started at an obese status at age 20 , with an average BMI of 32.4, and remained in the obese BMI range until age 55 . The proportion of obese women in this group was greater than $70 \%$ at the age of 20 . Conversely, the prevalence of being underweight or normal weight was negligible after age 40 .

The impacts of the risk factors, TVCs, age, and food insecurity on the BMI trajectories were examined based on the generalized model which included all covariates for women and men, respectively. As shown in Table 3, compared to Non-Aboriginal women, Aboriginal women had higher odds of being in the N-OV, OV-OB, and OB-UP groups, rather than the N-S group ( $\mathrm{OR}=2.6,7.1$, and 12.2, resp.). However, it was found that education was not a significant risk factor. In addition, increased years of being physically active, smoking, and drinking were associated with lowering the BMI trajectory in each group for women. Food insecurity, increased years of living with low income, being employed, and rural living were associated with raising the BMI trajectory in each group for women. Furthermore, a younger age was associated with raising the BMI trajectory in the N-OV group but lowering the trajectory in the OB-UP group. There were no significant associations found in the N-S and OV-OB groups of women.

For men, the GMP of the N-S BMI trajectory group was $31.7 \%$. The N-S trajectory remained in the normal weight range between ages 20 and 55 years.

Approximately $43.6 \%$ of men belonged to the N-OV group. The $\mathrm{N}-\mathrm{OV}$ trajectory started in the normal weight range at age 20 , with an average $\mathrm{BMI}$ of 23.8 , then progressed to the overweight range, and remained overweight until age 55 . Within the N-OV group, $18 \%$ of the men had a BMI of less than 25.0 by age 30 , and the majority of men in this group were overweight after age 40 . The proportion of obese men in this group increased steadily after age 40 , but this proportion did not exceed $25 \%$ at any age.

Approximately $20 \%$ of men belonged in the OV-OB BMI trajectory group. This trajectory started with an overweight status at age 20, with an average BMI of 26.8, increased to reach obese class I, and kept increasing but remained in the BMI range of obese class I. About $75 \%$ of men in the OV-OB group were overweight at age 20, but the prevalence of being overweight, without being obese, was negligible by age 55 . The prevalence of obesity in this group exceeded $85 \%$ after age 45 .

The GMP of the OB-UP group was $4.4 \%$. The OB-UP trajectory started at an obese status at age 20 , with an average BMI of 31.6, and remained in the obese BMI range until age 55. The proportion of obese men in this group was greater than $70 \%$ at the age of 20 . The prevalence of being underweight or normal weight was negligible after age 40 .

In men, neither education nor race/ethnicity was predictive of BMI trajectory group membership. Increased years of smoking was associated with lowering the BMI trajectory in each group for men. Increased years of being physically active and increased years of drinking alcohol were associated with lowering the BMI trajectory in all groups of men, with the exception of the N-S group. Increased years of living with low income was associated with raising the BMI trajectory in the OV-OB and OB-UP groups but was associated with lowering the trajectory in the N-S group. Increased years of employment was associated with raising the BMI trajectory in the N-S, N-OV, and OV-OB groups, while it was associated with lowering the trajectory in the OBUP group. Furthermore, increased years of rural living was associated with raising the BMI trajectory in the N-S group 
TABLE 3: Risk factors for BMI trajectory group membership, and TVCs influencing trajectory level within each group, NPHS, 1994-2011.

\begin{tabular}{|c|c|c|c|c|}
\hline Predictors & $\begin{array}{l}\text { Women } \\
\text { odds }^{*}\end{array}$ & $P$ value & $\begin{array}{l}\text { Men } \\
\text { odds }\end{array}$ & $P$ value \\
\hline \multicolumn{5}{|l|}{ Aboriginal } \\
\hline $\mathrm{N}-\mathrm{S}$ & 1.0 & - & 1.0 & - \\
\hline $\mathrm{N}-\mathrm{OV}$ & 2.6 & 0.047 & 1.4 & 0.43 \\
\hline $\mathrm{OV}-\mathrm{OB}$ & 7.1 & $<0.0001$ & 1.2 & 0.67 \\
\hline OB-UP & 12.2 & $<0.0001$ & 0.9 & 0.89 \\
\hline \multicolumn{5}{|c|}{ Not graduated from high school } \\
\hline N-S & 1.0 & - & 1.0 & - \\
\hline $\mathrm{N}-\mathrm{OV}$ & 1.03 & 0.84 & 0.9 & 0.43 \\
\hline $\mathrm{OV}-\mathrm{OB}$ & 1.05 & 0.74 & 1.2 & 0.15 \\
\hline OB-UP & 0.93 & 0.77 & 0.9 & 0.72 \\
\hline & Women & & Men & \\
\hline TVCs & $\begin{array}{c}\text { Alter in BMI trajectory per unit } \\
\text { change in TVCs }\end{array}$ & $P$ value & $\begin{array}{c}\text { Alter in BMI trajectory per unit } \\
\text { change in TVCs }\end{array}$ & $P$ value \\
\hline \multicolumn{5}{|l|}{ Years of PA } \\
\hline N-S & -0.03 & 0.003 & 0.04 & $<0.001$ \\
\hline $\mathrm{N}-\mathrm{OV}$ & -0.06 & $<0.001$ & -0.03 & 0.008 \\
\hline OV-OB & -0.05 & 0.005 & -0.10 & $<0.001$ \\
\hline OB-UP & -0.09 & $<0.001$ & -0.36 & $<0.001$ \\
\hline \multicolumn{5}{|c|}{ Years of smoking } \\
\hline N-S & -0.05 & $<0.001$ & -0.05 & $<0.001$ \\
\hline $\mathrm{N}-\mathrm{OV}$ & -0.04 & 0.001 & -0.04 & $<0.001$ \\
\hline $\mathrm{OV}-\mathrm{OB}$ & -0.03 & 0.045 & -0.05 & $<0.001$ \\
\hline OB-UP & -0.09 & $<0.001$ & -0.20 & $<0.001$ \\
\hline \multicolumn{5}{|c|}{ Years of regular drinking } \\
\hline $\mathrm{N}-\mathrm{S}$ & -0.02 & 0.029 & 0.02 & 0.053 \\
\hline $\mathrm{N}-\mathrm{OV}$ & -0.10 & $<0.001$ & -0.03 & 0.003 \\
\hline OV-OB & -0.16 & $<0.001$ & -0.12 & $<0.001$ \\
\hline OB-UP & -0.30 & $<0.001$ & -0.10 & $<0.001$ \\
\hline \multicolumn{5}{|c|}{ Years of living with low income } \\
\hline N-S & 0.05 & $<0.001$ & -0.07 & $<0.001$ \\
\hline $\mathrm{N}-\mathrm{OV}$ & 0.07 & $<0.001$ & 0.02 & 0.064 \\
\hline OV-OB & 0.10 & $<0.001$ & 0.10 & $<0.001$ \\
\hline OB-UP & 0.26 & $<0.001$ & 0.16 & $<0.001$ \\
\hline \multicolumn{5}{|c|}{ Years of employment } \\
\hline N-S & 0.08 & $<0.001$ & 0.04 & 0.003 \\
\hline $\mathrm{N}-\mathrm{OV}$ & 0.10 & $<0.001$ & 0.07 & $<0.001$ \\
\hline $\mathrm{OV}-\mathrm{OB}$ & 0.17 & $<0.001$ & 0.11 & $<0.001$ \\
\hline OB-UP & 0.18 & $<0.001$ & -0.20 & $<0.001$ \\
\hline \multicolumn{5}{|c|}{ Years of rural living } \\
\hline N-S & 0.08 & $<0.001$ & 0.03 & 0.002 \\
\hline $\mathrm{N}-\mathrm{OV}$ & 0.10 & $<0.001$ & -0.01 & 0.354 \\
\hline $\mathrm{OV}-\mathrm{OB}$ & 0.17 & $<0.001$ & -0.07 & $<0.001$ \\
\hline OB-UP & 0.18 & $<0.001$ & -0.20 & $<0.001$ \\
\hline \multicolumn{5}{|c|}{ Food insecurity $^{* *}$} \\
\hline N-S & 0.85 & $<0.001$ & 0.26 & 0.002 \\
\hline $\mathrm{N}-\mathrm{OV}$ & 2.09 & $<0.001$ & 0.78 & $<0.001$ \\
\hline $\mathrm{OV}-\mathrm{OB}$ & 3.18 & $<0.001$ & 1.37 & $<0.001$ \\
\hline OB-UP & 4.23 & $<0.001$ & 0.61 & 0.019 \\
\hline \multicolumn{5}{|c|}{ Age cohort ${ }^{* * *}$} \\
\hline N-S & 0.17 & 0.129 & 0.23 & 0.039 \\
\hline $\mathrm{N}-\mathrm{OV}$ & 0.78 & $<0.001$ & 0.96 & $<0.001$ \\
\hline $\mathrm{OV}-\mathrm{OB}$ & 0.10 & 0.620 & 1.57 & $<0.001$ \\
\hline OB-UP & -1.74 & $<0.001$ & 5.91 & $<0.001$ \\
\hline
\end{tabular}

${ }^{*}$ Relative adjusted odds ratios for membership in each trajectory using the N-S group as the reference class.

** Latent variable which records the most probable long-term food insecurity status of individuals.

*** Age cohorts: $20-29$ years at baseline versus $30-39$ years at baseline.

$\mathrm{N}-\mathrm{S}$, normal stable group; N-OV, normal weight to overweight group; OV-OB, overweight to obesity group; OB-UP, obesity-up group; and TVCs, the short for time-varying covariates. 
in men but was associated with lowering the trajectory in the $\mathrm{OV}-\mathrm{OB}$ and OB-UP groups. However, no associations were found in the N-OV group. In addition, a younger age and food insecurity were associated with raising the BMI trajectory in all of the groups for men.

\section{Discussion}

The results provide evidence of the heterogeneity of BMI trajectories among the population of young to middle-aged Canadian adults. The identified BMI trajectory groups of $\mathrm{N}-\mathrm{S}, \mathrm{N}-\mathrm{OV}, \mathrm{OV}-\mathrm{OB}$, and OB-UP are consistent with the findings of previous research on the US population of adults $[25,26]$. In addition, the effects of lifestyle behaviours and sociodemographic characteristics on the BMI trajectories were explored. Gender differences were observed between these associated factors and BMI trajectories. This is similar to the results found in [27] on body weight trajectories of baseline BMI groups of normal weight, overweight, and classes I, II, and III obese individuals. Thus, compared with previous research, this study provides additional information on the impact of lifestyle factors on BMI changes over time.

This study found that Aboriginal women were more likely to be in the higher BMI groups (N-OV, OV-OB, and OBUP) compared to the N-S group. This is consistent with the evidence from previous studies $[12,56]$. For instance, $\mathrm{Ng}$ et al. established that Aboriginal Canadians had a higher BMI trajectory with increasing rates of BMI and higher BMI peak values compared to their non-Aboriginal counterparts [12]. Thus, Aboriginal populations, specifically women, would benefit from healthy lifestyle programs or interventions to help prevent and reduce excess body weight during adulthood.

Educational attainment, defined by graduation from high school, was not a significant predictor of BMI trajectory group membership in either women or men. This finding is in agreement with the results from some previous studies [44, 57], but not with others [25, 33, 45]. For example, Ostbye et al. reported that increased years of education was associated with a lower BMI trajectory within each group [25]. This inconsistency may be due to the fact that in this study educational attainment was considered as a risk factor, whereas Ostbye et al. considered educational attainment as a TVC. A limitation of our study is that we only distinguished Aboriginal and Non-Aboriginal populations. Previous research has indicated that educational attainment may or may not affect BMI in different ethnic/racial groups [58].

It was found that increased years of tobacco smoking was associated with lowering the BMI trajectory in each group for both women and men. Nonnemaker et al. considered smoking as a risk factor and found that current smokers, aged 12-23 years, were more likely to be in the low-to-moderate risk or moderate-to-high risk groups of becoming obese compared to nonsmokers. The difference in findings may be due to the different age groups studied as well as the different ways that smoking was measured. In this study, smoking was measured in years and considered as a TVC, whereas smoking status was measured once and considered as a time-constant variable in the study by Nonnemaker et al. [22]. In another BMI trajectory study, the researchers utilized CGM and found that smoking was associated with a lower BMI at baseline and a slower rate of change over time [59]. Likewise, a pooled cross-sectional study suggested that smoking was associated with a lower BMI [60]. Furthermore, various other studies have determined that, on average, smokers weigh less than nonsmokers $[50,61]$. A strength of this study is that the data were obtained from respondents aged 20-55 years which are suggested to be the most common years of smoking [62]. A limitation of this study is that heavy smokers and light smokers were not distinguished due to unavailable data on smoking in the NPHS. According to previous research, the amount of smoking, whether heavy or light, may play an important role in BMI changes over time [61].

This study suggests that increased years of being physically active during leisure time is associated with lowering the BMI trajectory in women and men, except for the N-S group in men. This finding implies that individuals who maintain moderate or active physical activity during leisure time can reduce the likelihood of weight gain during adulthood. This is consistent with the documented inverse relationship between body weight and physical activity, particularly for women $[46,49]$. This finding provides evidence for including physical activity in any intervention targeted towards weight management.

It was found that increased years of regularly drinking alcohol was associated with lowering the BMI trajectory in each group in women and men, except for the N-S group of men. There is evidence that long-term, daily alcohol consumption can affect macronutrient absorption, thereby decreasing overall energy intake [63]. The results are consistent with the findings in a recent prospective cohort study with a 12.9-year follow-up, which reported that women with light to moderate alcohol consumption were less likely to gain weight or become obese. This association was consistent even after adjusting for other behavioural and clinical factors [64]. Though many researchers agree that alcohol consumption tends to increase the energy content of a person's dietary intake, there is no clear evidence on the association between drinking alcohol and gaining weight. Previous research findings on the possible associations between obesity and alcohol consumption are mixed [51]. The inconsistent findings may be because the majority of previous studies did not consider the potential heterogeneity of BMI changes over time in the studied population. There are some limitations with the findings in this study due to unavailable data in the NPHS. Firstly, different types of alcoholic beverages were not distinguished. Previous research has suggested that various types of alcoholic beverages may affect body weight differently. For example, light to moderate wine intake may protect against weight gain, whereas heavy intake of spirits, such as vodka, rum, or whiskey, may lead to weight gain [51]. Secondly, "regular drinking" was defined as drinking more than once per month which fails to consider the dose-dependent relationship between drinking alcohol and weight changes. Thirdly, this study did not distinguish between drinking patterns, such as binge drinking, which may play a determinant 
role in the association between obesity and alcohol intake [65].

The results suggest that increased years of living with low income was associated with raising the BMI trajectory in each group of women but the same association was only observed in the OV-OB and OB-UP groups for men. This finding is consistent with a study which reported that living with low income was more strongly associated with an increased risk of obesity in women as compared to men [66]. However, Ostbye et al. reported that increased years of living in poverty was not associated with trajectory change in any BMI trajectory group after considering other covariates. This inconsistent finding may be due to the difference in income level considered as well as the fact that Ostbye et al. did not model BMI trajectories separately for women and men.

The findings suggest that increased years of living in a rural area is associated with raising the BMI trajectory in all groups of women but was only observed in the normal weight group of men. This may be due to the fact that, in rural areas, men are often the "bread winners" and work outside the home whereas women are more likely to be homemakers. This finding is supported by evidence from a US study where obesity has been found to be more prevalent in rural areas compared with urban areas [48]. This may be associated with limited resources, limited access to health care systems, limited access to healthy food, and limited access to physical activity facilities or programs in rural areas $[48,67]$. Therefore, rural areas require more focus in the development public health programs and strategies to deal with the obesity epidemic, especially for women.

It was found that a younger age at baseline (20-29 years versus 30-39 years) was associated with raising the BMI trajectory in all groups for men but not for women. Previous studies have found that younger individuals tend to have faster increasing rates of BMI during adulthood $[12,25,68]$.

Increased years of being employed was associated with raising the BMI trajectory in all groups for both women and men, except for the OB-UP group in men. It has been proposed that employees may consume energy dense foods due to work related stress and that this can lead to weight gain $[69,70]$. Thus, reducing work related stress through health and wellness programs in the workplace may help to manage weight gain. For employers, this may then improve productivity and decrease time off due to obesity related illness.

The results suggest that food insecurity is associated with raising the BMI trajectory in all groups for both women and men. This finding is consistent with previous research that has found that individuals who were food insecure were more likely to be overweight or obese $[47,71,72]$. It should be noted that much Canadian research investigating food security/insecurity has used the food security module developed for the Canadian Community Health Survey (CCHS) which defines food security/insecurity somewhat differently than the present investigation [73]. Another limitation of this study pertaining to food security/insecurity is that the NPHS did not have information on people who were homeless or who live on Aboriginal reserves. These populations may be the most vulnerable to food insecurity in Canada. The findings in this study contribute new evidence by using latent variables to capture subgroups that are more likely to experience food insecurity. Policies and programs are needed to address food insecurity in Canada to ensure that all Canadians have access to safe and healthy food.

One important strength of this study is that LCGM was adopted to detect the heterogeneity in BMI change patterns. In addition, a representative sample from the NPHS was used. Furthermore, many potential factors that may contribute to obesity, such as lifestyle behaviours and sociodemographic characteristics, were investigated.

However, several limitations should be noted. One limitation is that individuals who had less than four BMI records in the NPHS were excluded from the analyses. The respondents included differed from those that were excluded in terms of ethnic/racial background, education level, mean BMI, family income, employment status, rural residence, smoking, alcohol consumption, and food security. This may limit the ability to generalize the findings to the entire population. Potential selection bias may exist; however, it was likely small in this study given the use of imputed BMI data from Statistics Canada and the modelling methods of LCGM. The results were produced using all available data including the individuals who had four or more BMI records even if they dropped out the survey. The cumulative attrition rate to cycle nine of the included group was $45.8 \%$.

Another possible limitation is that BMI was calculated using self-reported heights and weights which may introduce inaccuracies. BMI has also been suggested to be an unreliable tool as a measure of a healthy body weight. This is because BMI does not take into account muscle mass or fat mass, body shape or type, age, gender, and ethnicity/race. All of these factors may affect body weight and therefore a high $\mathrm{BMI}$ is not necessarily indicative of excess body fat. A more accurate measure of excess body fat, and therefore a healthy weight, would be body fat percentage measured using skin fold calipers; however, no longitudinal data of measured body fat could be found. This may be an interesting area for future research.

\section{Conclusion}

The four increasing BMI trajectory groups identified in this study show heterogeneity in the BMI changes of young to middle-aged Canadian adults over time. These findings help to identify those who are at an increased risk of becoming overweight or obese during adulthood. Gender differences were observed between the associated factors and BMI trajectories.

This study has identified factors associated with obesity that may allow clinicians and policy makers to allocate resources and tailor programs for specific groups of the Canadian population. Rather than utilize generic "one-size fits all" approaches to address the issue of obesity, it may be worthwhile for policy makers to examine the unique characteristics of specific populations, their BMI trajectories, and the risk factors associated with those trajectories as they develop prevention and/or intervention initiatives. 
Considering the unique BMI trajectories and associated risk factors in specific populations may lead to more successful outcomes. Also given that gender disparities were observed in associated factors of excess body weight, potential policies and solutions aimed at obesity prevention or weight loss may be more effective if they are designed with gender as a consideration. Further, the association of living with low income and food insecurity with BMI trajectory implies that social programs should be developed to make healthy food accessible to low income families in combatting the obesity epidemic. Long term campaigns to promote physical activity in rural areas should be initiated.

\section{Abbreviations \\ BMI: Body Mass Index \\ LCGM: Latent class growth modelling \\ CGM: Conventional growth modelling \\ NPHS: National Population Health Survey \\ GMP: Group membership probability \\ AvePP: Average posterior probability of group membership \\ TVC: Time-varying covariate \\ PA: Physical activity.}

\section{Conflict of Interests}

The authors of this paper indicated no competing interests.

\section{Authors' Contribution}

Meng Wang conducted the data analysis and drafted the first version of the paper. Yanqing Yi designed the study and provided advice on statistical modelling and interpretation of the results. Barbara Roebothan, Peizhong Peter Wang, and Guang Sun helped design the study and provided insight regarding interpretation of the results from the perspectives of nutrition, epidemiology, and metabolism, respectively. Jennifer Colbourne edited the paper and helped interpret the results. Victor Maddalena helped design the study and identified implications of the findings. All of the authors participated in the writing/revision of the paper and provided final approval.

\section{Acknowledgment}

The authors acknowledge the funding support from the Dean's Innovation Fund from Memorial University's Medical Research Endowment Fund.

\section{References}

[1] L. K. Twells, D. M. Gregory, J. Reddigan, and W. K. Midodzi, "Current and predicted prevalence of obesity in Canada: a trend analysis," CMAJ Open, vol. 2, no. 1, pp. E18-E26, 2014.

[2] J. C. Peters, H. R. Wyatt, W. T. Donahoo, and J. O. Hill, "From instinct to intellect: the challenge of maintaining healthy weight in the modern world," Obesity Reviews, vol. 3, no. 2, pp. 69-74, 2002.
[3] D. P. Guh, W. Zhang, N. Bansback, Z. Amarsi, C. L. Birmingham, and A. H. Anis, "The incidence of co-morbidities related to obesity and overweight: a systematic review and meta-analysis," BMC Public Health, vol. 9, article 88, 2009.

[4] J. P. Block, Y. He, A. M. Zaslavsky, L. Ding, and J. Z. Ayanian, "Psychosocial stress and change in weight among US adults," American Journal of Epidemiology, vol. 170, no. 2, pp. 181-192, 2009.

[5] A. Botoseneanu and J. Liang, "Latent heterogeneity in longterm trajectories of body mass index in older adults," Journal of Aging and Health, vol. 25, no. 2, pp. 342-363, 2013.

[6] J. Lynch and G. D. Smith, "A life course approach to chronic disease epidemiology," Annual Review of Public Health, vol. 26, pp. 1-35, 2005.

[7] S. Dare, D. F. Mackay, J. P. Pell, and K. Matsuo, "Relationship between smoking and obesity: a cross-sectional study of 499,504 middle-aged adults in the UK general population," PLoS ONE, vol. 10, no. 4, Article ID e0123579, 2015.

[8] B. K. Jacobsen, I. Njølstad, I. Thune, T. Wilsgaard, M.-L. Løchen, and H. Schirmer, "Increase in weight in all birth cohorts in a general population: the Tromsø study, 1974-1994," Archives of Internal Medicine, vol. 161, no. 3, pp. 466-472, 2001.

[9] C. E. Lewis, D. R. Jacobs Jr., H. McCreath et al., "Weight gain continues in the 1990s: 10-year trends in weight and overweight from the CARDIA study. Coronary Artery Risk Development in Young Adults," American Journal of Epidemiology, vol. 151, no. 12, pp. 1172-1181, 2000.

[10] C. E. Lewis, D. E. Smith, D. D. Wallace, O. D. Williams, D. E. Bild, and D. R. Jacobs Jr., "Seven-year trends in body weight and associations with lifestyle and behavioral characteristics in Black and White young adults: the CARDIA study," American Journal of Public Health, vol. 87, no. 4, pp. 635-642, 1997.

[11] T. J. J. Sheehan, S. DuBrava, L. M. DeChello, and Z. Fang, "Rates of weight change for black and white Americans over a twenty year period," International Journal of Obesity, vol. 27, no. 4, pp. 498-504, 2003.

[12] C. Ng, P. N. Corey, and T. K. Young, "Divergent body mass index trajectories between Aboriginal and non-Aboriginal Canadians 1994-2009-an exploration of age, period, and cohort effects," American Journal of Human Biology, vol. 24, no. 2, pp. 170-176, 2012.

[13] M. S. Setia, A. Quesnel-Vallee, M. Abrahamowicz, P. Tousignant, and J. Lynch, "Convergence of body mass index of immigrants to the Canadian-born population: evidence from the National Population Health Survey (1994-2006)," European Journal of Epidemiology, vol. 24, no. 10, pp. 611-623, 2009.

[14] L. B. Sherar, J. C. Eisenmann, P. D. Chilibeck et al., "Relationship between trajectories of trunk fat mass development in adolescence and cardiometabolic risk in young adulthood," Obesity, vol. 19, no. 8, pp. 1699-1706, 2011.

[15] T. Abarin, Y. Y. Wu, N. Warrington, S. Lye, C. Pennell, and L. Briollais, "The impact of breastfeeding on FTO-related BMI growth trajectories: an application to the Raine pregnancy cohort study," International Journal of Epidemiology, vol. 41, no. 6, pp. 1650-1660, 2012.

[16] X. Wen, K. Kleinman, M. W. Gillman, S. L. Rifas-Shiman, and E. M. Taveras, "Childhood body mass index trajectories: modeling, characterizing, pairwise correlations and sociodemographic predictors of trajectory characteristics," $B M C$ Medical Research Methodology, vol. 12, article 38, 2012. 
[17] A. S. Bryk and S. W. Raudenbush, "Application of hierarchical linear models to assessing change," Psychological Bulletin, vol. 101, no. 1, pp. 147-158, 1987.

[18] J. J. McArdle and D. Epstein, "Latent growth curves within developmental structural equation models," Child Development, vol. 58, no. 1, pp. 110-133, 1987.

[19] D. S. Nagin, Group-Based Modelling of Development, Harvard University Press, Cambridge, Mass, USA, 2005.

[20] D. S. Nagin and C. L. Odgers, "Group-based trajectory modeling in clinical research," Annual Review of Clinical Psychology, vol. 6, pp. 109-138, 2010.

[21] D. S. Nagin, "Analyzing developmental trajectories: a semiparametric, group-based approach," Psychological Methods, vol. 4, no. 2, pp. 139-157, 1999.

[22] J. M. Nonnemaker, A. A. Morgan-Lopez, J. M. Pais, and E. A. Finkelstein, "Youth BMI trajectories: evidence from the NLSY97," Obesity, vol. 17, no. 6, pp. 1274-1280, 2009.

[23] C. A. Magee, P. Caputi, and D. C. Iverson, "Identification of distinct body mass index trajectories in Australian children," Pediatric Obesity, vol. 8, no. 3, pp. 189-198, 2013.

[24] L. E. Pryor, R. E. Tremblay, M. Boivin et al., "Developmental trajectories of body mass index in early childhood and their risk factors: an 8-year longitudinal study," Archives of Pediatrics and Adolescent Medicine, vol. 165, no. 10, pp. 906-912, 2011.

[25] T. Ostbye, R. Malhotra, and L. R. Landerman, "Body mass trajectories through adulthood: results from the National Longitudinal Survey of Youth 1979 Cohort (1981-2006)," International Journal of Epidemiology, vol. 40, no. 1, pp. 240-250, 2011.

[26] E. A. Finkelstein, T. Ostbye, and R. Malhotra, "Body mass trajectories through midlife among adults with class I obesity," Surgery for Obesity and Related Diseases, vol. 9, no. 4, pp. 547553, 2013.

[27] R. Malhotra, T. Østbye, C. M. Riley, and E. A. Finkelstein, "Young adult weight trajectories through midlife by body mass category," Obesity, vol. 21, no. 9, pp. 1923-1934, 2013.

[28] M. N. Kuchibhatla, G. G. Fillenbaum, W. E. Kraus, H. J. Cohen, and D. G. Blazer, "Trajectory classes of body mass index in a representative elderly community sample," Journals of Gerontology, Series A: Biological Sciences and Medical Sciences, vol. 68, no. 6, pp. 699-704, 2013.

[29] A. Botoseneanu and J. Liang, "Social stratification of body weight trajectory in middle-age and older Americans: results from a 14-year longitudinal study," Journal of Aging and Health, vol. 23, no. 3, pp. 454-480, 2011.

[30] R. Kanter and B. Caballero, "Global gender disparities in obesity: a review," Advances in Nutrition, vol. 3, no. 4, pp. 491498, 2012.

[31] N. A. Christakis and J. H. Fowler, "The spread of obesity in a large social network over 32 years," The New England Journal of Medicine, vol. 357, no. 4, pp. 370-379, 2007.

[32] W. Yang, T. Kelly, and J. He, "Genetic epidemiology of obesity," Epidemiologic Reviews, vol. 29, no. 1, pp. 49-61, 2007.

[33] L. McLaren, "Socioeconomic status and obesity," Epidemiologic Reviews, vol. 29, no. 1, pp. 29-48, 2007.

[34] L. D. Howe, R. Patel, and B. Galobardes, "Commentary: tipping the balance: wider waistlines in men but wider inequalities in women," International Journal of Epidemiology, vol. 39, no. 2, pp. 404-405, 2010.

[35] G. Jones-Johnson, W. R. Johnson, and N. Frishman, "Race and gender differences in obesity and disease," Sociology Mind, vol. 4, no. 3, pp. 233-241, 2014.
[36] A. M. El-Sayed, P. Scarborough, and S. Galea, "Unevenly distributed: a systematic review of the health literature about socioeconomic inequalities in adult obesity in the United Kingdom," BMC Public Health, vol. 12, article 18, 2012.

[37] B. M. Popkin, "Does global obesity represent a global public health challenge?" American Journal of Clinical Nutrition, vol. 93, no. 2, pp. 232-233, 2011.

[38] S. L. Gortmaker, B. A. Swinburn, D. Levy et al., "Changing the future of obesity: science, policy, and action," The Lancet, vol. 378, no. 9793, pp. 838-847, 2011.

[39] Statistics Canada, National Population Health Survey Household Component, Cycle 1 (1994/1995) to 9 (2010/2011), Longitudinal Documentation, 2012.

[40] S. Galbraith, J. Bowden, and A. Mander, "Accelerated longitudinal designs: an overview of modelling, power, costs and handling missing data," Statistical Methods in Medical Research, 2014.

[41] S. C. Duncan, T. E. Duncan, and H. Hops, "Analysis of longitudinal data within accelerated longitudinal designs," Psychological Methods, vol. 1, no. 3, pp. 236-248, 1996.

[42] P. Clarke, P. M. O’Malley, L. D. Johnston, and J. E. Schulenberg, "Social disparities in BMI trajectories across adulthood by gender, race/ethnicity and lifetime socio-economic position: 1986-2004," International Journal of Epidemiology, vol. 38, no. 2, pp. 499-509, 2009.

[43] D. E. Chiriboga, Y. Ma, W. Li et al., "Gender differences in predictors of body weight and body weight change in healthy adults," Obesity, vol. 16, no. 1, pp. 137-145, 2008.

[44] K. Ball and D. Crawford, "Socioeconomic status and weight change in adults: a review," Social Science and Medicine, vol. 60, no. 9, pp. 1987-2010, 2005.

[45] S. Hermann, S. Rohrmann, J. Linseisen et al., "The association of education with body mass index and waist circumference in the EPIC-PANACEA study," BMC Public Health, vol. 11, article $169,2011$.

[46] J. Cranfield, Factors Influencing the Body Mass Index of Adults in Canada, Consumer and Market Demand Agricultural Policy Research Network, Edmonton, Canada, 2007.

[47] L. Pan, B. Sherry, R. Njai, and H. M. Blanck, "Food insecurity is associated with obesity among US adults in 12 states," Journal of the Academy of Nutrition and Dietetics, vol. 112, no. 9, pp. 14031409, 2012.

[48] J. E. Jackson, M. P. Doescher, A. F. Jerant, and L. G. Hart, "A national study of obesity prevalence and trends by type of rural county," Journal of Rural Health, vol. 21, no. 2, pp. 140-148, 2005.

[49] B. Sternfeld, H. Wang, C. P. Quesenberry Jr. et al., "Physical activity and changes in weight and waist circumference in midlife women: findings from the study of women's health across the nation," American Journal of Epidemiology, vol. 160, no. 9, pp. 912-922, 2004.

[50] D. Canoy, N. Wareham, R. Luben et al., "Cigarette smoking and fat distribution in 21,828 British men and women: a populationbased study," Obesity Research, vol. 13, no. 8, pp. 1466-1475, 2005.

[51] C. Sayon-Orea, M. A. Martinez-Gonzalez, and M. BesRastrollo, "Alcohol consumption and body weight: a systematic review," Nutrition Reviews, vol. 69, no. 8, pp. 419-431, 2011.

[52] B. L. Jones, D. S. Nagin, and K. Roeder, "A SAS procedure based on mixture models for estimating developmental trajectories," Sociological Methods \& Research, vol. 29, no. 3, pp. 374-393, 2001. 
[53] H. Andruff, N. Carraro, A. Thompson, and P. Gaudreau, "Latent class growth modelling: a tutorial," Tutorials in Quantitative Methods for Psychology, vol. 5, no. 1, pp. 11-24, 2009.

[54] P. J. Clarke, P. M. O’Malley, J. E. Schulenberg, and L. D. Johnston, "Midlife health and socioeconomic consequences of persistent overweight across early adulthood: Findings from a national survey of American adults (1986-2008)," American Journal of Epidemiology, vol. 172, no. 5, pp. 540-548, 2010.

[55] C. Winship and L. Radbill, "Sampling weights and regression analysis," Sociological Methods \& Research, vol. 23, no. 2, pp. 230-257, 1994.

[56] M. Tjepkema, R. Wilkins, S. Senécal, E. Guimond, and C. Penney, "Mortality of Métis and registered Indian adults in Canada: an 11-year follow-up study," Health Reports, vol. 20, no. 4, pp. 31-51, 2009.

[57] S. A. French, R. W. Jeffery, J. L. Forster, P. G. McGovern, S. H. Kelder, and J. E. Baxter, "Predictors of weight change over two years among a population of working adults: the Healthy Worker Project," International Journal of Obesity and Related Metabolic Disorders, vol. 18, no. 3, pp. 145-154, 1994.

[58] T. T. Lewis, S. A. Everson-Rose, B. Sternfeld, K. Karavolos, D. Wesley, and L. H. Powell, "Race, education, and weight change in a biracial sample of women at midlife," Archives of Internal Medicine, vol. 165, no. 5, pp. 545-551, 2005.

[59] S. K. Kahng, R. E. Dunkle, and J. S. Jackson, "The relationship between the trajectory of body mass index and health trajectory among older adults: multilevel modeling analyses," Research on Aging, vol. 26, no. 1, pp. 31-61, 2004.

[60] I. A. Rossi, V. Rousson, B. Viswanathan, and P. Bovet, "Gender and socioeconomic disparities in BMI trajectories in the Seychelles: a cohort analysis based on serial population-based surveys," BMC Public Health, vol. 11, article 912, 2011.

[61] A. Chiolero, D. Faeh, F. Paccaud, and J. Cornuz, "Consequences of smoking for body weight, body fat distribution, and insulin resistance," The American Journal of Clinical Nutrition, vol. 87, no. 4, pp. 801-809, 2008.

[62] Centers for Disease Control and Prevention (CDC), "Cigarette smoking among adults-United States, 2006," Morbidity and Mortality Weekly Report, vol. 56, no. 44, pp. 1157-1161, 2007.

[63] R. K. Foster and H. E. Marriott, "Alcohol consumption in the new millennium-weighing up the risks and benefits for our health," Nutrition Bulletin, vol. 31, no. 4, pp. 286-331, 2006.

[64] L. Wang, I.-M. Lee, J. E. Manson, J. E. Buring, and H. D. Sesso, "Alcohol consumption, weight gain, and risk of becoming overweight in middle-aged and older women," Archives of Internal Medicine, vol. 170, no. 5, pp. 453-461, 2010.

[65] E. Lukasiewicz, L. I. Mennen, S. Bertrais et al., "Alcohol intake in relation to body mass index and waist-to-hip ratio: the importance of type of alcoholic beverage," Public Health Nutrition, vol. 8, no. 3, pp. 315-320, 2005.

[66] A. M. El-Sayed, P. Scarborough, and S. Galea, "Socioeconomic inequalities in childhood obesity in the United Kingdom: a systematic review of the literature," Obesity Facts, vol. 5, no. 5, pp. 671-692, 2012.

[67] J. D. Willms, M. S. Tremblay, and P. T. Katzmarzyk, "Geographic and demographic variation in the prevalence of overweight Canadian children," Obesity Research, vol. 11, no. 5, pp. 668-673, 2003.

[68] O. K. Caman, S. Calling, P. Midlöv, J. Sundquist, K. Sundquist, and S.-E. Johansson, "Longitudinal age-and cohort trends in body mass index in Sweden-a 24-year follow-up study," BMC Public Health, vol. 13, no. 1, article 893, 2013.
[69] A. Kouvonen, M. Kivimäki, S. J. Cox, T. Cox, and J. Vahtera, "Relationship between work stress and body mass index among 45,810 female and male employees," Psychosomatic Medicine, vol. 67, no. 4, pp. 577-583, 2005.

[70] T. I. J. van den Berg, L. A. M. Elders, B. C. H. de Zwart, and A. Burdorf, "The effects of work-related and individual factors the work ability index: a systematic review," Occupational and Environmental Medicine, vol. 66, no. 4, pp. 211-220, 2009.

[71] P. E. Wilde and J. N. Peterman, "Individual weight change is associated with household food security status," Journal of Nutrition, vol. 136, no. 5, pp. 1395-1400, 2006.

[72] K. S. Martin and A. M. Ferris, "Food insecurity and gender are risk factors for obesity," Journal of Nutrition Education and Behavior, vol. 39, no. 1, pp. 31-36, 2007.

[73] Household Food Insecurity in Canada: Overview, http://www .hc-sc.gc.ca/fn-an/surveill/nutrition/commun/insecurit/indexeng.php. 


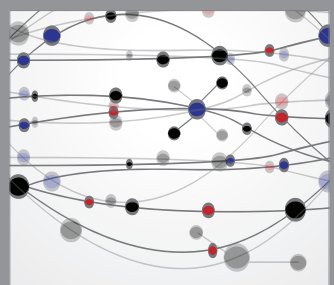

The Scientific World Journal
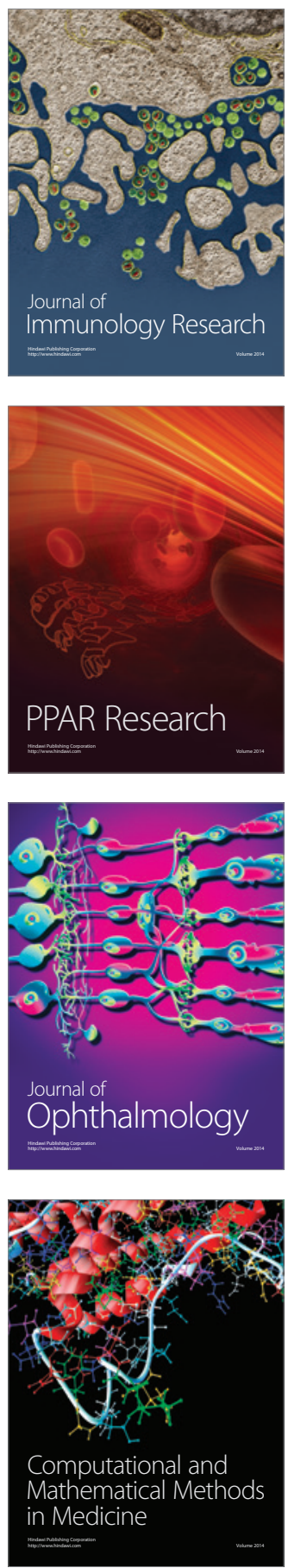

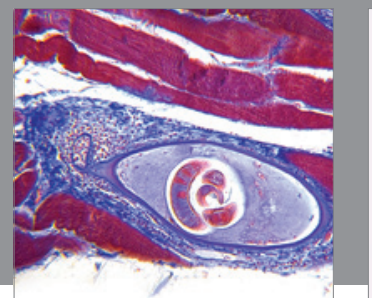

Gastroenterology

Research and Practice
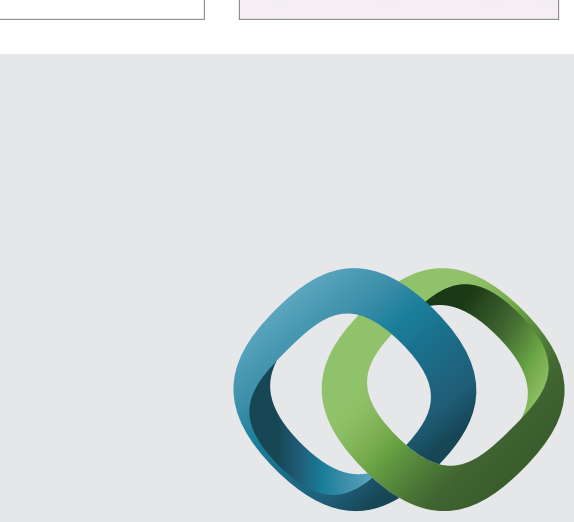

\section{Hindawi}

Submit your manuscripts at

http://www.hindawi.com
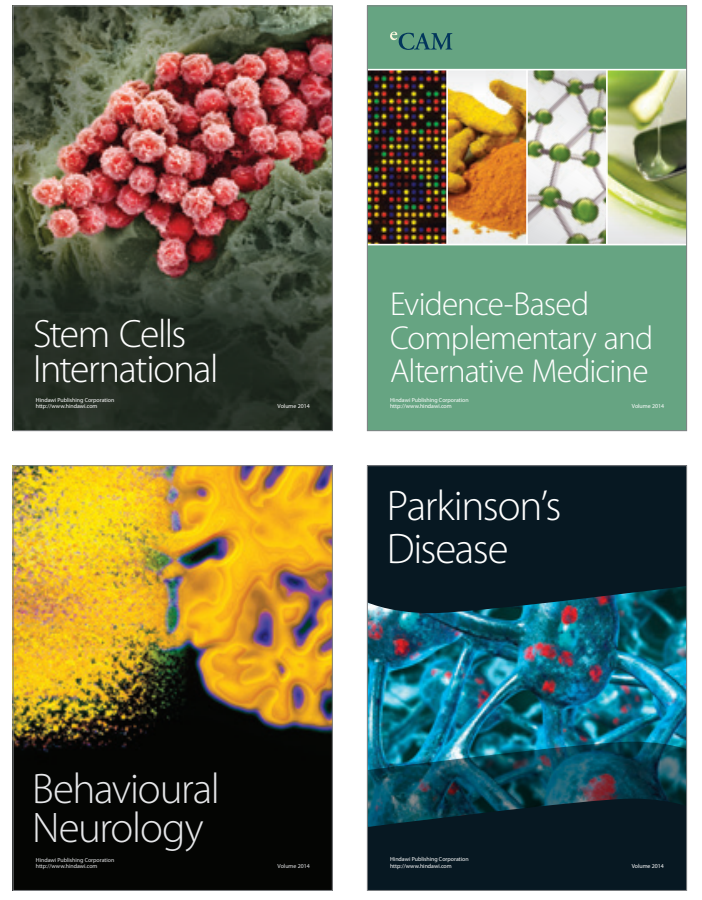
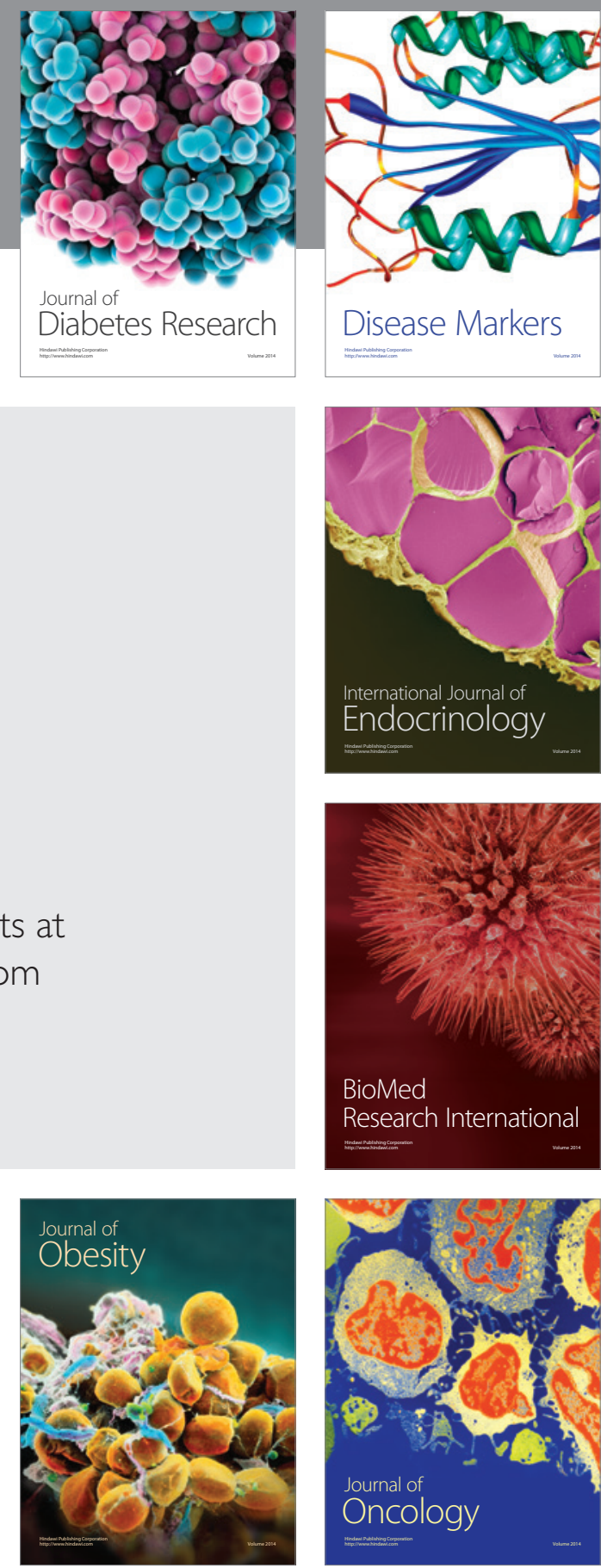

Disease Markers
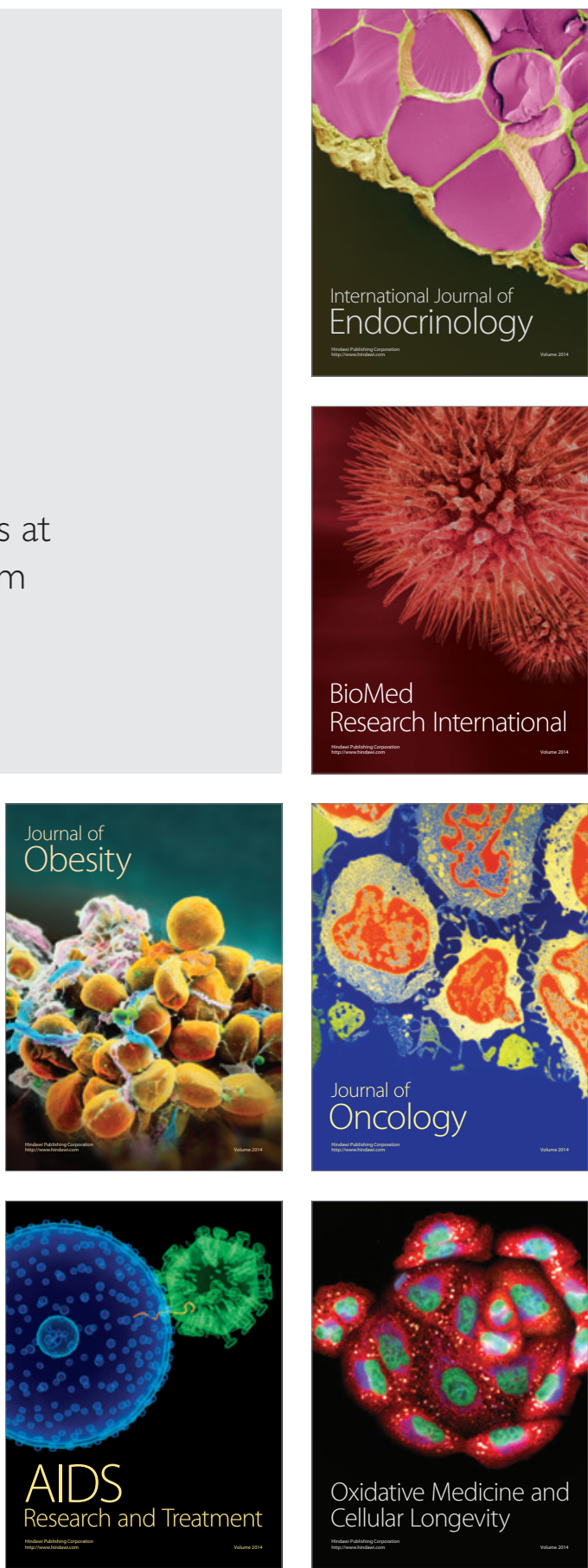